Editorial

\title{
Inventions 2018 Best Paper Award
}

\author{
Inventions Editorial Office
}

MDPI AG, St. Alban-Anlage 66, 4052 Basel, Switzerland; inventions@mdpi.com

Received: 2 November 2018; Accepted: 2 November 2018; Published: 2 November 2018

check for updates

We are pleased to announce the second "Inventions Best Paper Award" for 2018. Nominations, chosen from all papers published in 2017, were made by the Editorial Board. Following review by the Editorial Board, the three top-voted research articles as follows, in no particular order, have each won the "Inventions Best Paper Award" for 2018:

\section{Shantha Gamini Jayasinghe, Lasantha Meegahapola, Nuwantha Fernando, Zheming Jin and Josep} M. Guerrero

Review of Ship Microgrids: System Architectures, Storage Technologies and Power Quality Aspects Inventions 2017, 2(1), 4; doi:10.3390/inventions2010004

Available online: https:/ / www.mdpi.com/2411-5134/2/1/4

With the ever-growing demand for emission reduction and fuel efficiency improvement, sustainable energy sources are becoming an integral part of maritime electrical power systems. As a result, electrical power systems in future ships or offshore platforms will consist of various sources and loads forming islanded microgrids. Similar to land-based microgrids, the challenge here will be matching sources and load dynamics while keeping the primary control objectives, such as voltage and/or frequency within a predefined range. Power electronic converters and energy storage systems play a crucial role in this matching process. Nevertheless, while playing a crucial role, power electronic converters could introduce waveform distortions and other power quality issues, especially at high power levels. Passive filtering is a commonly used technique for reducing such distortions, which requires bulky capacitors and inductors. Active filtering is emerging as an alternative, which could be realized even within the same interfacing converter of the energy storage system. This paper investigates recent developments in these areas and provides readers with a critical review of power quality issues, energy storage technologies and strategies that could be used to improve the power quality in ship microgrids.

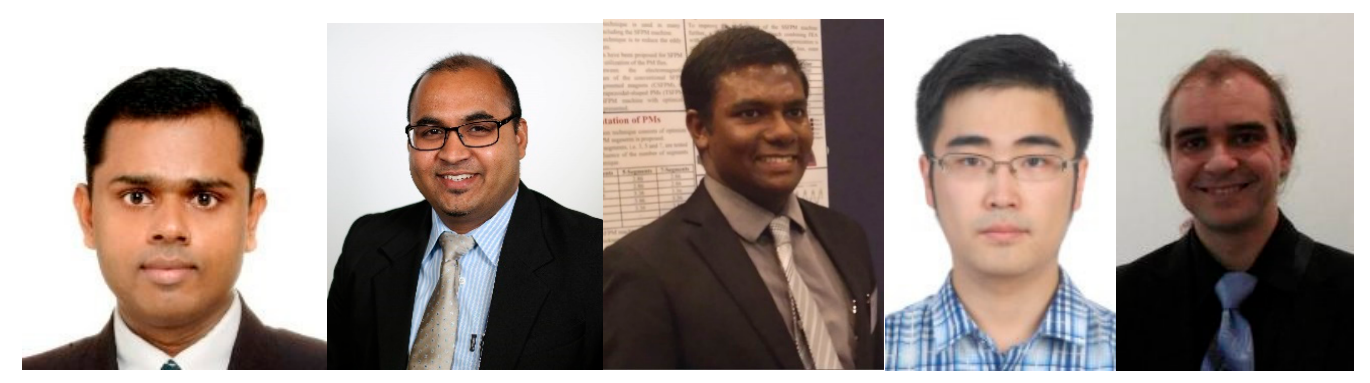

Shantha Gamini Jayasinghe, Lasantha Meegahapola, Nuwantha Fernando, Zheming Jin and Josep M. Guerrero (from left to right).

\footnotetext{
Georgiana Balaban, George Cristian Lazaroiu, Virgil Dumbrava and Catalina Alexandra Sima Analysing Renewable Energy Source Impacts on Power System National Network Code Inventions 2017, 2(3), 23; doi:10.3390/inventions2030023

Available online: https://www.mdpi.com/2411-5134/2/3/23
} 
The authors analyze the impact of renewable energy sources integrated into the Romanian power system. The reduction of power demand led to difficulties in integrating the renewable energy sources into the power system (network reinforcements), as well as challenges concerning the balance between production and consumption. Following the excess of certain shares of energy mix, intermittent renewable energy sources require the expansion of networks, storage, back-up capacities and efforts for flexible consumption. The authors observe that the absence of these requirements may lead to an overloaded grid and to the inability of using renewable energy sources. To highlight the difficulty of connecting some significant installed capacities, like wind power plants and photovoltaic installations, the authors present a case study for the Dobrogea area that has the largest installed capacity from renewable energy sources in operation. This power system area is connected to the rest of the country through three highly loaded transmission lines. The authors performed simulations for the considered real network located in Romania and, in particular, for the case of an important bus where $600 \mathrm{MW}$ from wind power plants (WPP) are connected. In order to obtain secure operation of the power system, a WPP of $210 \mathrm{MW}$ (the largest one), connected at a very loaded bus, may be disconnected. The authors highlight the necessity of power system reinforcement actions in order to overcome network problems determined by these volatile sources.

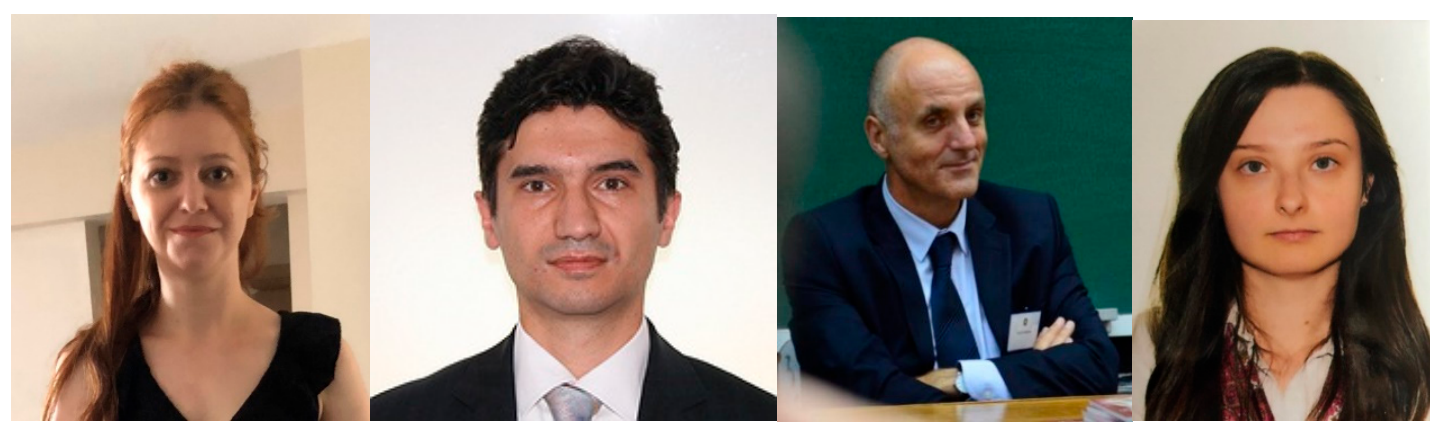

Georgiana Balaban, George Cristian Lazaroiu, Virgil Dumbrava and Catalina Alexandra Sima (from left to right).

\section{Kunal Mondal}

Recent Advances in the Synthesis of Metal Oxide Nanofibers and Their Environmental Remediation Applications

Inventions 2017, 2(2), 9; doi:10.3390/inventions2020009

Available online: https://www.mdpi.com/2411-5134/2/2/9

Photocatalytic oxidation could be proven as an efficient alternative method for the oxidation and elimination of recalcitrant organic species under specific conditions for the management of agricultural and industrial pollutants in wastewater.

Nearly two hundred of the latest papers on nanostructured semiconductor photocatalysts and their synthesis and oxidation-mediated environmental applications are cited in this review. There are several metal oxide photocatalysts available; however, among them, metal oxide nanofiber morphologies are reasonably stable and could retain structural integrity while employed under ultraviolet (uv), visible, or solar light towards continuous applications such as environmental remediation, bacterial decontamination, and air purification etc.

There are many routes available for the synthesis of metal oxide photocatalysts and electrospinning is the one which is the most efficient among all of these for the fabrication of oxide nanofibers. Countless polymers, along with compatible precursors for metal oxides, have been efficaciously electrospun into nanosized fibers lately.

It was found that photocatalytic oxidation using metal oxides has tremendously enhanced the ability to degrade organic pollutants; however, the high cost of reagents, availability of energy sources and the higher bandgap energy of semiconductors are the main issues against efficient photocatalytic 
pollutant removal. These problems could be addressed by the use of solar irradiation and superior nanomorphology of metal oxide photocatalysts. Adjusting electronic, optical, and physicochemical properties of the metal oxides can ensure the efficient degradation of pollutants.

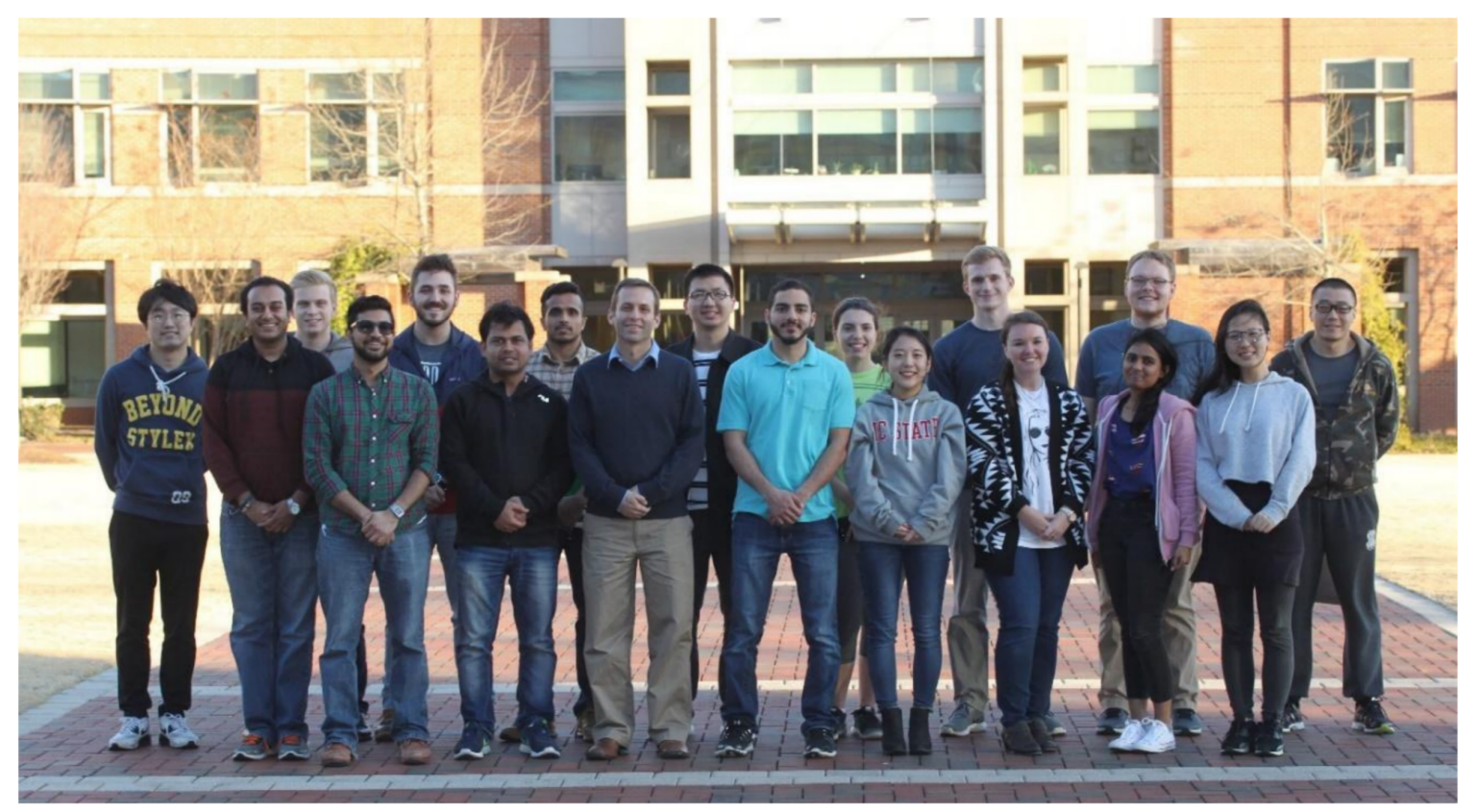

Research group at North Carolina State University. Dr. Kunal Modal is in the front row, 4th from the left.

We believe that these three exceptional papers make valuable contributions to Inventions and the scientific research field. On behalf of the Inventions Editorial Board, we would like to congratulate these teams for their excellent work. A certificate will be given to each of them.

We would like to take this opportunity to thank all the nominated research groups of the above exceptional papers for their contributions to Inventions, and to thank the Inventions Editorial Board for voting and helping with this "Best Paper Award".

The Editorial Board and Editorial Staff at Inventions is committed to meeting the needs of the science community by providing useful and timely reviews of all manuscripts submitted and providing an open access journal for your results. Please consider submitting your work to Inventions, and we look forward to announcing your paper as an Inventions Best Paper in the future.

Prize Awarding Committee

Inventions Editorial Board

(C) 2018 by the author. Licensee MDPI, Basel, Switzerland. This article is an open access article distributed under the terms and conditions of the Creative Commons Attribution (CC BY) license (http://creativecommons.org/licenses/by/4.0/). 